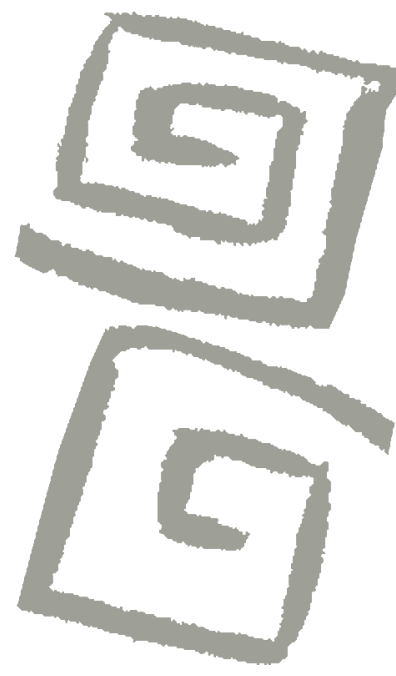

\title{
¿Por qué los médicos no utilizan en forma temprana la insulinización en pacientes con diabetes mellitus tipo 2?: Un estudio cualitativo en una ciudad mexicana
}

\author{
Why don't doctors use early insulinization therapy in \\ patients with diabetes mellitus type 2?: A qualitative \\ approach in a Mexican city
}

Roberto Lagunes-Córdoba' ${ }^{1}$ Isaac Galindo-Guevara², Atalia Castillo Reyes ${ }^{3}$, Citlalli Romero-Aparicio ${ }^{4}$, Francisco Javier Rosas-Santiago ${ }^{5}$

\begin{abstract}
${ }^{1}$ Doctor en Ciencias. Investigador de tiempo completo, Instituto de Investigaciones Psicológicas, Universidad Veracruzana. Veracruz, México. $\bowtie$ iD

${ }^{2}$ Licenciado en Psicología. Estudiante, Especialización en Estudios de Opinión, Facultad de Psicología, Universidad Veracruzana. Veracruz, México. $\triangle$ (iD)

${ }^{3}$ Maestra en Salud Pública. Estudiante de Doctorado en Psicología, Instituto de Investigaciones Psicológicas, Universidad Veracruzana. Xalapa, Veracruz, México. $\triangle$ (iD)

${ }^{4}$ Licenciada en Psicología. Becaria, Facultad de Psicología, Universidad Veracruzana. Xalapa Veracruz, México. $\Delta$ iD

${ }^{5}$ Doctor en Ciencias. Investigador de tiempo completo, Instituto de Investigaciones Psicológicas, Universidad Veracruzana. Xalapa, Veracruz, México. $\triangle$ iD
\end{abstract}

RESUMEN La terapia de insulinización temprana ha demostrado ser un auxilio eficaz para mejorar el control a largo plazo y la calidad de vida de pacientes con diabetes mellitus tipo 2. Sin embargo, tanto los pacientes como los médicos presentan barreras al uso de esta alternativa terapéutica. El presente estudio utiliza un abordaje cualitativo para explorar las barreras a la insulinización temprana que tiene el personal médico del sector público en la ciudad de Xalapa, Veracruz, México. Entre los años 2015 y 2016, se realizaron entrevistas en profundidad a médicos generales y especialistas que brindaban atención primaria a pacientes con diabetes mellitus tipo 2. Las entrevistas transcritas fueron analizadas para extraer y esquematizar las categorías y subcategorías de las barreras del personal de salud, las cuales se agruparon en tres categorías que fueron ejemplificadas con extractos del discurso de los participantes: barreras propias del personal médico, barreras que surgen en la relación médico-paciente, y obstáculos institucionales. Se discuten los posibles usos del esquema obtenido, así como algunas propuestas de solución generadas por los participantes del estudio.

PALABRAS CLAVES Diabetes Mellitus Tipo 2; Cuerpo Médico de Hospitales; Terapéutica; México.

\begin{abstract}
Early insulinization therapy is regarded as an efficient aid to improve long term control and quality of life in patients with diabetes mellitus type 2 (DM2). Nevertheless, both patients and medical staff confront barriers in using this therapeutic tool. This study employs a qualitative approach to explore the barriers to early insulinization among medical staff from the public sector in the city of Xalapa, Veracruz, México. Between 2015 and 2016, in-depth interviews were conducted with general and specialist physicians offering primary health care to patients with DM2. The transcribed interviews were analyzed to extract and organize categories and subcategories of barriers among medical staff. These barriers were then grouped into three categories and exemplified with interview excerpts: barriers coming from the medical staff itself, barriers emerging from the doctor-patient interaction, and institutional barriers. Uses for the classification obtained are discussed, as are some of the solutions proposed by study participants.
\end{abstract}

KEY WORDS Diabetes Mellitus Type 2; Hospital Medical Staff; Therapeutics; Mexico. 


\section{INTRODUCCIÓN}

La diabetes mellitus tipo 2 (DM2) es un trastorno metabólico crónico caracterizado por concentraciones elevadas y persistentes de glucosa en la sangre como consecuencia de deficiencias o alteraciones en la acción de la insulina en el organismo ${ }^{(1)}$. Es una enfermedad multiorgánica y plurimetabólica, que se caracteriza por una baja respuesta de los tejidos corporales a la insulina que secreta el páncreas, o porque este órgano es incapaz de secretar la insulina necesaria para la absorción de la glucosa. Los pacientes que la padecen pueden ser situados en un continuo en el cual, puede predominar la resistencia a la insulina con relativamente poca deficiencia de su secreción, o predomina la deficiencia de secreción con algún grado de resistencia a la insulina ${ }^{(2)}$. Hasta ahora no se ha encontrado un tratamiento eficaz para esta enfermedad, y su prevalencia va en aumento en todo el mundo. En el año 2000 se presentaron 171 millones de casos a nivel mundial, y la cifra se ha incrementado a 422 millones para el año $2014^{(3,4)}$.

La DM2 es una enfermedad de causas múltiples que, si no se trata adecuadamente, ocasiona complicaciones de salud graves como: infarto del corazón, ceguera, falla renal, amputación de extremidades inferiores y muerte prematura ${ }^{(5)}$. Los costos físicos, psicológicos, económicos y sociales de la enfermedad son cada vez mayores y sus comorbilidades incluyen daño microvascular, con sus complicaciones asociadas (pie diabético, retinopatía, daño renal), isquemia cardiaca e infarto ${ }^{(6)}$.

En México, la DM2 ha alcanzado proporciones alarmantes, convirtiéndose en un problema de salud pública. Actualmente es la primera causa de hospitalización, y la tasa de mortalidad por diabetes se incrementó del $43,3 \%$ al $53,2 \%$ por cada 100.000 habitantes entre 1998 y $2002^{(7)}$. De acuerdo con la Encuesta Nacional en Salud y Nutrición (ENSANUT) de 2012, la proporción de adultos con diagnóstico médico de DM2 fue del $9,2 \%$, lo que muestra un incremento importante en comparación con el 5,8\% reportado en la ENSANUT 2000 y con el $7 \%$ de la ENSANUT 2006 ${ }^{(5)}$. Esto repercute en altos costos para el sistema de salud, pérdidas por incapacidad y deterioro de la calidad de vida de los pacientes ${ }^{(6,7,8)}$. A partir de 1990, la DM2 apareció dentro del cuadro de principales causas de muerte en México, y hoy es la principal causa de defunción en todo el país ${ }^{(9)}$.

A nivel regional, el estado de Veracruz es la tercera entidad más poblada de México ${ }^{(10)}$, y de acuerdo con las datos de la ENSANUT 2012 también ocupa el tercer lugar en prevalencia de DM2, con el 10,7\% de adultos que han sido diagnosticados ${ }^{(5)}$. En el estado de Veracruz se reportaron 27.824 casos nuevos de diabetes en 2012, de los cuales la jurisdicción de Xalapa se encuentra dentro de las tres entidades que reportaron el mayor número de casos.

Debido a la naturaleza crónica de la enfermedad y la ausencia de una cura definitiva, el tratamiento de la diabetes es muy complejo y multifactorial. Las metas del tratamiento son claras y requieren que los pacientes cumplan con medidas terapéuticas basadas en criterios estrictos ${ }^{(11)}$. El logro de estas metas requiere de un manejo dietético apoyado con ejercicio, control de la glucemia con automonitoreo (especialmente en pacientes que reciben insulina), y el uso de antidiabéticos orales $o$ insulina ${ }^{(11,12,13)}$. Las guías de la American Diabetes Association (ADA), de la European Association for the Study of Diabetes (EASD), de la Asociación Latinoamericana de Diabetes (ALAD), y del National Institute for Health and Care Excellence (NICE) recomiendan que, cuando el paciente presenta una clínica estable, la función pancreática está conservada y puede darse el control metabólico mediante inmunosensibilizadores y conservadores de la función pancreática, sin que exista riesgo de descompensación moderada a severa, se utilicen antidiabéticos orales. La primera elección es la metformina, y como segunda elección inhibidores de la dipeptidil peptidasa-4 (DPP-4) o glitazonas en diversas combinaciones. Cuando estos tratamientos fallan o no pueden ser tolerados por el 
paciente, se recomienda comenzar la terapia con insulina ${ }^{(11,14,15,16)}$.

Además de los tratamientos ya mencionados, se ha ido produciendo un consenso en la literatura que afirma que, aunque no sea la terapéutica de elección en las guías modernas, salvo en casos y excepciones señaladas $^{(15)}$, el uso temprano de la insulina (antes o de manera concurrente a los antidiabéticos orales) favorece el control de la DM2 a largo plazo $^{(17,18,19,20)}$. Se ha demostrado que la terapéutica con insulina es el tratamiento que disminuye en mayor proporción los niveles de hemoglobina glucosilada, y se considera que es segura y efectiva para el tratamiento en pacientes diabéticos, siempre que se siga el esquema de tratamiento apropiado ${ }^{(17,21,22)}$.

A pesar de estas evidencias, el uso de la insulina suele ser pospuesto hasta 10 a 15 años después del diagnóstico de $\mathrm{DM} 2^{(23)}$. Esto se debe a que el problema de iniciar la terapia con insulina (insulinización) es muy complejo, y abarca múltiples factores del paciente, de los médicos, y del mismo sistema de salud(24). Cuando estos factores obstaculizan el uso de la terapia con insulina y limitan la adherencia a este tipo de terapia se constituyen en barreras a la insulinización ${ }^{(25)}$. Existen diversas investigaciones en las que se documentan gran cantidad de obstáculos y barreras al uso de la insulina, y la mayor parte de los estudios, especialmente en México y América Latina, se ha enfocado en los pacientes. Las barreras de los pacientes abarcan situaciones de rechazo y falta de disposición para el uso de la insulina ${ }^{(26,27)}$, ansiedad $^{(25)}$, falta de conocimiento y sentimientos negativos asociados a su uso ${ }^{(28,29)}$, la atribución de síntomas físicos, como el aumento de peso o la ceguera ${ }^{(30)}$, el uso de terapias alternativas, o la opinión de la familia ${ }^{(31,32)}$.

En lo que se refiere al personal de salud, el problema está menos estudiado. Pero se ha documentado que, aunque existen normas y modelos de atención para el paciente diabético y amplias evidencias científicas del beneficio de la terapia temprana con insulina, los médicos se resisten a prescribir insulina $^{(33)}$. Las creencias de los médicos y sus actitudes retrasan el inicio de la terapia con insulina y algunas de estas son similares a las de los pacientes. Entre las barreras a la insulinización que se han reportado en el personal de salud están: la percepción de la baja eficacia de la insulina, el temor a que los pacientes presenten hipoglucemia, el aumento de peso con el riesgo cardiovascular asociado, la inercia terapéutica del personal de salud, el temor al incumplimiento del tratamiento por parte del paciente, la historia natural de la enfermedad y los problemas que tienen relación con el sistema de salud y que influyen en que el paciente y el profesional logren los objetivos ${ }^{(33,34,35)}$.

En el modelo de salud que está vigente en México desde 2015, la disposición de los médicos hacia la insulinización es crucial, ya que en muchos casos son ellos los que proponen su uso, o determinan si el paciente debe utilizarla o no. En México, la terapéutica de la diabetes se basa en la Norma Oficial Mexicana NOM-015-SSA2-2010 para la prevención, el control y el tratamiento de la diabetes meIlitus, y en el Modelo Integral de Atención al Paciente ${ }^{(36,37)}$. Ambos documentos establecen de manera explícita la responsabilidad del médico sobre el tratamiento, prevención y control de la diabetes, y en qué momento y de qué manera utilizar las estrategias farmacológicas y no farmacológicas, incluyendo la terapéutica con insulina. Sin embargo, exceptuando la revisión de Lerman ${ }^{(33)}$ y el trabajo de Ávalos-García et al. ${ }^{(35)}$, no hemos encontrado estudios en México sobre las barreras que el personal médico pueda presentar hacia la terapia de insulinización temprana. Esta falta de información tiene graves consecuencias ya que, de acuerdo con la Encuesta Nacional de Salud 2012, solo el 13\% de los pacientes diagnosticados con DM2 reciben terapia con insulina, sola o acompañada de hipoglucemiantes orales ${ }^{(38)}$.

Si consideramos las ventajas de la insulinización temprana y el papel central del médico en el tratamiento y la educación del paciente diabético, el conocimiento de las barreras que estos pueden presentar hacia la terapia con insulina podría utilizarse para crear estrategias para vencerlas, y contribuir a que los pacientes con DM2 sean canalizados 
apropiadamente para el uso de una herramienta terapéutica potencialmente valiosa. Debido a lo anterior y al hecho de que se carece de información sobre las barreras a la terapia de insulinización temprana en el personal médico en México, incluyendo ciudades con alta prevalencia de DM2, hemos planteado la realización de un estudio exploratorio, de naturaleza cualitativa, para generar conocimiento sobre las posibles barreras a la insulinización por parte del personal médico en un contexto mexicano, y establecer un intento de clasificación de estas barreras que pueda coadyuvar a su posible resolución. De manera adicional, los médicos participantes generaron ideas y posibles estrategias de solución para algunas de las barreras encontradas en este estudio.

\section{METODOLOGÍA}

El presente estudio fue de naturaleza cualitativa y exploratoria. Se analizó el contenido de entrevistas en profundidad realizadas a médicos del primer nivel de atención con experiencia en la atención de pacientes con DM2 de la Ciudad de Xalapa, estado de Veracruz, en México. En el sector público del sistema de salud mexicano, el personal médico del primer nivel tiene la responsabilidad de brindar la atención inicial a pacientes con padecimientos que no requieren atención especializada, y son los que toman el enfoque preventivo y el control inicial de pacientes con DM2. Este tipo de personal suele ser médico general o especialista en medicina familiar. El control de pacientes descompensados, con comorbilidades o complicaciones asociadas corresponde a médicos del segundo nivel de atención, entre los que se encuentran los médicos internistas o diabetólogos ${ }^{(36)}$.

Los participantes fueron reclutados informalmente en diferentes instituciones del sector salud del estado de Veracruz, por medio de referencias primarias o secundarias. Los médicos reclutados proporcionan atención a través del sector público, que es financiado por el gobierno federal y estatal.

Tras haber realizado un contacto inicial, se les explicó en qué consistía el estudio y se les proporcionó un formato de consentimiento informado. En caso de estar de acuerdo en participar, se acordó un sitio de reunión con condiciones adecuadas para la grabación de las entrevistas, a una hora conveniente para los investigadores y los participantes. Se obtuvieron 18 grabaciones, de las cuales 10 correspondían a médicos generales, 6 a médicos especialistas en medicina familiar, y 2 a médicos internistas. Todos ellos tenían un mínimo de 3 años de experiencia en la atención a pacientes diabéticos y estaban activos en la atención de ese tipo de pacientes hasta el momento de la entrevista. Tres de ellos combinaban la atención a pacientes con un cargo administrativo al interior de su institución de adscripción.

Con el fin de lograr información amplia, detallada, contextualizada y útil para la comprensión de la temática de investigación, se realizaron entrevistas en profundidad con los participantes del estudio. Se hizo un esfuerzo particular para establecer un rapport adecuado y establecer un clima de confianza desde el principio de la entrevista. En estas condiciones, la entrevista posibilitaba que los participantes expresaran sus opiniones sinceras, y que establecieran un intercambio que permitiera explorar a fondo las creencias, actitudes y motivaciones de los entrevistados respecto a la temática planteada ${ }^{(39)}$.

En las entrevistas se buscó obtener una descripción de las barreras que impiden que los médicos consideren la terapia de insulinización temprana para pacientes con DM2. La guía de entrevista utilizada contaba con cuatro preguntas:

1. Relate brevemente su experiencia en el tratamiento, control y seguimiento de pacientes con diabetes mellitus tipo 2.

2. ¿Cuáles son los principales retos y obstáculos a los que se enfrenta el paciente con diabetes mellitus tipo 2 ?

3. ¿Cuáles son las barreras que tienen los pacientes con diabetes mellitus tipo 2 a la terapia con insulina? 
4. ¿Cuáles son las barreras que tienen los médicos hacia el uso de la terapia con insulina por sus pacientes con diabetes mellitus tipo 2 ?

Aunque no se hizo una clasificación previa de las barreras, se tomaron en cuenta los antecedentes de la literatura para plantear un esquema de análisis pertinente (Figura 1). Con el fin de obtener un panorama lo más completo posible, se recolectó información más allá del punto de saturación, manteniendo la expectativa de obtener información nueva en sucesivas entrevistas. La saturación fue evidente al cabo de ocho entrevistas, variando solo en detalles accesorios y muy específicos de la situación personal de los participantes. La suficiencia de la información se decidió de común acuerdo entre los integrantes del grupo de investigación ${ }^{(40)}$.

Las entrevistas tuvieron duraciones de entre 30 y 60 minutos, fueron transcritas

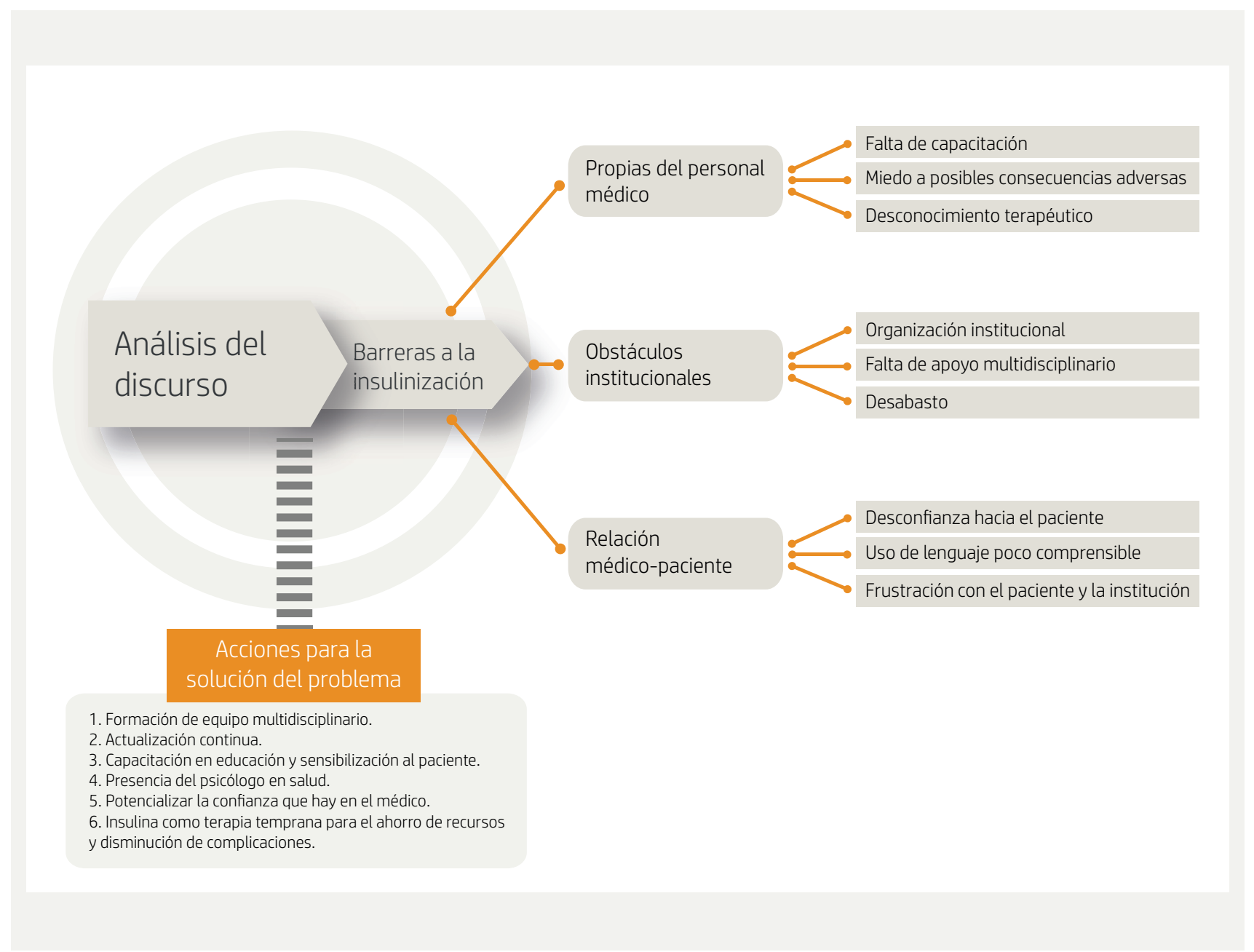

Figura 1. Mapa de análisis del discurso a partir de transcripciones de entrevistas realizadas a personal médico que atiende a pacientes con diabetes mellitus 2 en la Ciudad de Xalapa, estado de Veracruz, México. 2015 y 2016.

Fuente: Elaboración propia. 
por cuatro integrantes del equipo de investigación, y a partir de las cuatro versiones se integró una versión común que fue utilizada para los análisis. La codificación de la información se llevó a cabo de manera manual, por cada uno de los miembros del equipo y, una vez terminada, se llevaron a cabo reuniones para consensuar las categorías encontradas. Las categorías iniciales en las que se codificaron los datos fueron: barreras propias del personal médico, barreras surgidas en la relación médico-paciente y obstáculos institucionales. Durante el análisis y discusión conjunta surgieron subcategorías derivadas de las anteriores.

Finalmente, en el análisis del discurso se encontraron propuestas del mismo personal médico para afrontar estas problemáticas, las cuales serán desarrolladas y discutidas en una sección posterior.

\section{Análisis de la información}

El análisis se llevó a cabo buscando fragmentos representativos de las categorías planteadas tras la revisión de la bibliografía. Asimismo, por medio de la técnica de las comparaciones constantes se buscó material consistente y representativo para plantear nuevas categorías y subcategorías ${ }^{(41,42)}$. Las categorías y subcategorías encontradas, junto con sus relaciones, se representaron en forma esquemática para facilitar su análisis e interpretación.

\section{Aspectos éticos}

La confidencialidad de los datos fue salvaguardada restringiendo el acceso a las grabaciones a cualquier persona que no fuera miembro del equipo de investigación del estudio. Los nombres de los participantes y de cualquier persona involucrada fueron omitidos de las preguntas, las transcripciones y cualquier registro impreso relacionado con la presente investigación. El estudio fue aprobado por el Comité de Investigación y Ética del Instituto de Investigaciones Psicológicas de la
Universidad Veracruzana, según resolución del día 4 de septiembre de 2015.

\section{RESULTADOS}

El material obtenido mediante el análisis y la transcripción de las entrevistas mostró una gran convergencia. A partir de la tercera entrevista, el material obtenido comenzó a presentar repeticiones, y para la octava entrevista comenzó a ser evidente la saturación de todas las subcategorías.

El esquema inicial de tres categorías fue complementado, toda vez que las categorías iniciales se dividieron a su vez en subcategorías con fuertes vínculos en común. A continuación, se discuten las categorías, con sus subcategorías y fragmentos de discurso representativos.

\section{Barreras propias del personal médico}

Esta categoría incluye barreras que son atribuibles a situaciones, conocimiento $y$ creencias propias de los médicos, independientemente de su relación terapéutica con algún paciente en particular. En el presente estudio encontramos: desconocimiento del valor de la insulinización temprana como opción terapéutica, la falta de capacitación en atención al paciente y el miedo a posibles consecuencias adversas para el paciente.

\section{Desconocimiento del valor de la insulinización temprana como opción terapéutica}

Algunos médicos, sobre todo los no especialistas, consideran que no tienen los conocimientos necesarios para utilizar la insulinización temprana con los pacientes:

...De manera personal, yo no conozco todas esas insulinas, pero sé que en su momento es oportuno hacer una cierta combinación... (Médica general, mujer) 
...muchos de los médicos no están familiarizados con la insulina ¿sí?; incluso a algunos les falla el hecho de calcular las dosis de insulina, las combinaciones, por eso es que prefieren que los vean otros médicos... (Médico general, hombre)

En general, los médicos familiares y los internistas consideraban que sabían manejar esquemas terapéuticos de insulinización temprana, pero señalaban que el desconocimiento de esos esquemas era un obstáculo importante para que otros colegas los utilizaran en sus pacientes.

...los médicos, sobre todo médicos que ya tienen mucho tiempo laborando, ya no tienen tantas ganas de seguirle, ni documentándose, y siguen usando tratamientos y terapéuticas que les enseñaron cuando estuvieron en la facultad. Entonces todo eso retrasa el inicio de la terapia con insulina... (Médica internista, mujer)

Esta barrera ha sido reportada por otros autores, y en esas investigaciones, los médicos no especialistas reconocen que dudan de su capacidad para manejar un régimen de insulinización temprana, y muchos de ellos prefieren canalizar al paciente a un segundo nivel; en cambio, aquellos con nivel de especialidad consideran que pueden utilizar un esquema de insulinización temprana beneficioso para sus pacientes ${ }^{(33,35,43)}$. Esta barrera por sí misma constituye un impedimento para que los pacientes puedan recibir esta opción terapéutica, ya que en muchas comunidades no existen especialistas de segundo nivel de atención, y los pacientes no serán canalizados a estos servicios.

\section{Falta de capacitación en atención al paciente}

Dado que la insulinización temprana requiere que el paciente aprenda todo lo relacionado con el manejo, cuidado y dosificación de la insulina; además de vencer sus previsibles barreras hacia este tipo de terapia, el personal médico debería estar capacitado para educar al paciente y ayudarle a vencer sus barreras hacia la insulinización. Sin embargo, los médicos generales y más de la mitad de los especialistas consideran que nunca aprendieron a hacer esto, y que la capacitación recibida fuera de sus estudios formales no resulta adecuada para ello:

...el médico definitivamente no está
capacitado para... para explicarle a un
paciente, para enseñarle. Estamos acos-
tumbrados o estamos... [duda al escoger
sus palabras] enseñados a que debemos
curarlos y no prevenirlos, es uno de
nuestros errores más grandes... (Médico
general, hombre)

...personalmente le digo que yo no sé cómo debo tratar a un paciente que me dice que le tiene miedo a los piquetes. Les puedo informar que no se van a quedar ciegos, o que pueden aprender a manejar las dosis para no tener bajones de glucosa, pero no puedo contra sus miedos... eh... abstractos, diría yo... (Médica internista, mujer)

Un aspecto importante es que la falta de capacitación en el manejo integral del paciente diabético no ha sido adecuadamente tratada en la bibliografía sobre barreras a la insulinización del personal médico. El enfoque sistémico familiar y el modelo de manejo integral del paciente contemplan el apoyo de la familia y del resto del personal de salud en la atención y el manejo del paciente diabético, pero en los documentos consultados no se señala con claridad qué estrategias puede utilizar el médico para capacitarse en aquellos aspectos del manejo del paciente que no domina. Este aspecto de la educación para el paciente es tan importante que requiere estudios posteriores.

\section{Miedo a posibles consecuencias adversas para el paciente}

Casi todos los participantes mencionaron este temor como un factor disuasorio para 
empezar la insulinización con sus pacientes, sea o no de manera temprana. En general, los temores principales que se reportan son hacia la ocurrencia de episodios de hipoglucemia, problemas cardiacos y en el caso de algunos médicos generales, el aumento de peso:

...con la insulina en cualquier momento puedes causar hipoglucemia o, en caso de que no le esté pasando bien la dosis, vas a tener un estado de hiperglucemia constante en el paciente; y muchos no se acostumbran... (Médico general, hombre)

...también tenemos el riesgo de que el paciente aumente de peso y se pueda descompensar más rápido, o tener problemas cardiacos... (Médica general, mujer)

Estos temores han sido documentados en diversos trabajos ${ }^{(33,34,44,45)}$, y parecen ser prevalentes a pesar de existir guías y consensos sobre el uso de la insulina, y la manera de prevenir las complicaciones del tratamiento. Es destacable el hecho de que los médicos especialistas, y sobre todo los familiares, enfatizaron que la hipoglucemia, aunque es el riesgo más peligroso y mejor documentado en la bibliografía, puede ser evitado o reducido si los médicos se toman el tiempo necesario para educar a los pacientes:

...el riesgo de hipoglucemia existe como una complicación del uso de insulina. Es por eso que cuando nosotros iniciamos la terapia de insulinización debemos prever que a ese paciente lo vamos a estar viendo de una manera más frecuente, incluso semanalmente, hasta que encontremos la dosis que lo mantiene. También... se les explica que ahí es donde la nutrición tiene mucho que ver, porque para el uso de la insulina... Bueno, necesitan llevar una adecuada alimentación, saber que tienen que llevar sus quintos o sus colaciones, y el momento en que se van a aplicar su insulina... (Médica internista, mujer)

\section{Barreras surgidas en la relación médico paciente}

Otro grupo de barreras surgen de manera específica en la relación del médico con los pacientes. Este tipo de barreras parece depender mucho de la manera en que ambos se perciben y se comunican. En esta categoría se han incluido como subcategorías: la desconfianza hacia el paciente, el uso de un lenguaje poco comprensible para el paciente, y la frustración que se genera durante la relación.

\section{Desconfianza hacia el paciente}

Parece existir un consenso entre los médicos acerca de que el paciente no va a entender o aplicar las recomendaciones, e incluso que podría engañar al médico deliberadamente sobre la manera en que está llevando la terapia con insulina:

...me ha pasado varias veces que parece que el paciente ya entendió, y le pido que me repita las indicaciones y no puede hacerlo. Ya doy por hecho que no me van a entender, y que voy a tener que explicar muchas veces el tratamiento a mis pacientes nuevos. (Médica general, mujer)

...he encontrado pacientes que juran que llevan su tratamiento, hasta que un día tienen un coma diabético o verificamos sus niveles de hemoglobina glucosilada, y descubrimos que no lo siguen desde hace meses... (Médico internista, hombre)

De acuerdo con lo encontrado en la bibliografía, una de las posibles consecuencias de esta desconfianza es que genera predisposiciones negativas del médico hacia el paciente, lo que se traduce en críticas y regaños constantes, y a crear una relación jerárquica en la cual el médico tiene la razón y el paciente debe seguir las indicaciones sin cuestionarlas ${ }^{(35)}$. Esto crea una situación paradójica: por un lado, la bibliografía enfatiza la 
necesidad de que el médico lleve al paciente a responsabilizarse por el control de su enfermedad y el apego a las indicaciones ${ }^{(35,44)}$. Pero en cambio, con su actitud, crea una situación asimétrica, haciendo al paciente un recipiente pasivo de sus indicaciones. $\mathrm{Y}$, encima de todo, lo critica por ello:

Las instituciones, el médico y el propio paciente tienen que saber más de su... de su enfermedad, ¿no? El hecho de que él mismo no exija a los médicos esa explicación. Se quedan con lo que les explican, con que el paracetamol les va a quitar el dolor, la insulina les va a bajar el azúcar, pero no preguntan más. Entonces eso genera también poca importancia por parte de los médicos, sobre su paciente. (Médico general, hombre)

\section{Uso de un lenguaje poco comprensible para el paciente}

Una gran parte de los médicos generales, e incluso algún especialista señaló que en ocasiones le era complicado hacerse entender con el paciente, utilizar un lenguaje en el que pudiera dar a entender sus indicaciones:

...nunca aprendes a bajar el nivel de lo que estás diciendo... eh... a otro tipo de población... (Médico general, hombre)

...esto es cuestión de comunicación, es algo que también nos han venido manejando los últimos años en la relación médico-paciente y en la adecuada comunicación; tanto verbal en lo que le puedo estar diciendo con palabras, y también lo que le puedo estar expresando con mis movimientos, mis gesticulaciones, con mi tono de voz. Porque le decía desde un principio, vamos a ponernos de acuerdo... (Médica familiar, mujer)

Por supuesto, es posible considerar que esta barrera es un aspecto más de la falta de educación del médico y, por lo tanto, un problema particular de él. Pero varios especialistas comentaron que aun cuando su formación les permitiese tener herramientas o un lenguaje apropiado para comunicarse con la mayoría de los pacientes, no estaban exentos de tener problemas para comunicarse con alguno de ellos.

\section{...tras 18 años de práctica ya no es tan frecuente, pero sí me topo con pacientes a los que no se les da entender la comu- nicación verbal. Tengo que buscar analogías, formas de decir las cosas... cambiar el orden de las palabras... E incluso así, me pasa que no tengo la certeza de que me hayan entendido siempre... (Médica internista, mujer)}

Este tema adquiere particular importancia, ya que cuando no existe una comunicación efectiva, el médico no puede tener garantías de que su paciente comprenda y efectúe sus indicaciones como es necesario. Es frecuente que el médico emplee terminología y un lenguaje poco asequible para el paciente, dificultando de este modo la comprensión de sus indicaciones. Parece que la comunicación se ve alterada en el momento en que el médico intenta dar ciertas indicaciones en un lenguaje poco comprensible, $y$ esto no permite que la relación médico-paciente se construya en conjunto para llevar el tratamiento. El aspecto de la comunicación ha sido documentado ampliamente en otras situaciones y en relación con otras enfermedades $^{(45)}$, pero lo ha sido menos en el contexto de la terapia con insulina ${ }^{(35)}$. Y dado el riesgo potencial de eventos de hipoglucemia, debería ser abordado en profundidad al investigar el contexto de las terapias de insulinización.

\section{Frustración}

Más de la mitad de los participantes mencionaron la frustración generada en la relación médico-paciente como una barrera hacia la insulinización, sea temprana o en momentos posteriores. La aparente falta de 
comprensión del paciente puede ser un factor que los haga no plantearse proporcionar una terapia de insulinización:

...Definitivamente tienes que tener
una tolerancia a la frustración alta y
saber que, no todos los pacientes son
médicos, y no todos van a entender a la
primera qué es lo que tienen que hacer.
Muchas veces ni siquiera a la quinta va a
aprender qué tienen que hacer. Entonces
tienes que estar repitiéndoselo en cada
consulta, preguntar en cada consulta,
aunque ya sean pacientes con muchos
años usando insulina... (Médica general,
mujer)

Además del trabajo de Ávalos-García et al.(35), no encontramos antecedentes sobre este sentimiento de frustración y su relación con la atención a los pacientes en literatura consultada. Pero algunos antecedentes relacionados con la actitud del médico hacia el paciente parecen hablar de emociones y situaciones que generan frustración, aunque no la mencionen explícitamente. Por ejemplo, se ha señalado que el médico tiene tendencia a regañar y criticar al paciente, y a crear una relación jerárquica en la cual él tiene la razón y el paciente debe seguir las indicaciones sin cuestionarlas; pero, por otra parte, estos mismos médicos se quejan de que los pacientes no se involucran en el control de su enfermedad ${ }^{(35)}$. Por otro lado, hay médicos que se refieren a la terapia de insulinización como una opción de castigo o como un último recurso, si el paciente no se apega a las indicaciones ${ }^{(22,33,44)}$.

La desconfianza hacia el paciente, expresada por los médicos y los problemas de comunicación que existen en la relación entre ambos, es un asunto que desde la perspectiva de Barsky ${ }^{(46)}$ corresponde a lo que denomina "paradoja de la salud". Esta paradoja consiste en que, en los modelos sanitarios del mundo moderno, ocurren situaciones que invisibilizan la importancia de la relación médico paciente en la práctica clínica. Puede considerarse que este fenómeno facilita que, a pesar del evidente progreso científico en el área médica, sea cada vez mayor el deterioro en la salud y el bienestar percibidos por la población. También, es posible que se presente una menor satisfacción por parte de los médicos en su quehacer, lo que causaría o incrementaría sus sentimientos de frustración. Todos estos factores pueden influirse entre sí, ya que la frustración del médico puede aumentar su resentimiento y la desconfianza hacia el paciente y esto, a su vez, cerrar las vías de comunicación con él. Con ello, las alternativas terapéuticas como la insulinización temprana podrían no plantearse, o incluso no ser consideradas por el médico. Este no es un asunto simple y justifica la tarea de desentrañar la lógica implícita en la comunicación médico paciente para mostrarla como un producto socialmente construido; es decir, con fuertes asociaciones entre los arreglos sociales y las condiciones de vida de ambos actores ${ }^{(47)}$.

La relación médico-paciente determina la puesta en marcha y aceptación del tratamiento en personas que padecen enfermedades crónico-degenerativas como la $\mathrm{DM} 2^{(48)}$. Los pacientes con diabetes prefieren un modelo de relación con sus médicos en el que los escuchen, empaticen con su situación, comprendan los problemas que implica para ellos el tratamiento, les transmitan ánimo y adapten sus recomendaciones a las circunstancias vitales y emocionales individuales ${ }^{(49)}$. Al respecto, los resultados de este estudio plantean la necesidad de enriquecer las investigaciones en cuanto a la relación médico-paciente, con el objetivo de mejorar la comprensión del papel del comportamiento humano en el proceso de salud-enfermedad y el perfeccionamiento de la práctica médica $^{(50)}$.

\section{Obstáculos institucionales}

El personal entrevistado es unánime al comentar que existen múltiples obstáculos a nivel institucional para llevar a cabo la terapia de insulinización. El exceso de consultas, el poco tiempo para atender cada paciente, $y$ la dificultad para contar con espacios ya han 
sido documentadas en otra investigación realizada en México ${ }^{(35)}$. Pero de manera particular, en el estado de Veracruz y en el momento en que se termina esta investigación, se viven situaciones de desabasto, no solo de insulina, sino de antidiabéticos orales y de los insumos necesarios para el diagnóstico y tratamiento de la diabetes. Estas situaciones dificultan brindar la atención adecuada o educar al paciente diabético, especialmente en esquemas de tratamiento complejos con insulina.

En esta categoría, encontramos tres grandes grupos de barreras relacionadas: organización institucional, desabasto y falta de apoyo multidisciplinario.

\section{Organización institucional}

Los profesionales reportan que existen obstáculos en el funcionamiento de la institución que dificultan la insulinización del paciente diabético. En concreto, hay políticas institucionales que privilegian el uso de otro tipo de terapias antes de llegar a la insulinización. La sobrecarga en el número de pacientes que deben atender y la estructura jerárquica propia del sistema de salud impiden plantear el uso de la insulinización temprana y sus ajustes correspondientes:

...aunque de manera muy personal le digo que el primer tratamiento que se debería dar es la insulina, las instituciones prefieren dar la insulina hasta que ya fallaron los otros dos tratamientos... (Médico general, hombre)

...traspolando a la institución, a veces es un poquito difícil. Tenemos tres mil diabéticos en la unidad y cada día se siguen diagnosticando. Por semana se están dando alrededor de 30 diagnósticos nuevos o más, y me estoy quedando corta. Son dos turnos más el fin de semana que se trabaja, son múltiples diagnósticos nuevos de diabetes. Entonces eso nos hace un total de más de seis mil diabéticos en la unidad para inicios de este año. Entonces, abarcarlos todos así es un poquito complejo, por la cantidad del personal que tenemos. (Médica familiar, mujer)

El exceso de consultas, el poco tiempo para atender cada paciente y la dificultad para contar con espacios ya han sido documentados al menos en otra investigación realizada en México ${ }^{(35)}$.

\section{Falta de apoyo multidisciplinario}

Los médicos, independientemente de su grado de especialización, perciben que hay situaciones con los pacientes que no pueden manejar solo y que se beneficiarían mucho de la intervención de especialistas de otras disciplinas en el manejo integral del paciente. Sin embargo, el apoyo multidisciplinario pocas veces está presente en sus lugares de trabajo $y$, en caso de que lo esté, es solamente para un número muy limitado de pacientes:

...en la Secretaría se manejan dos sistemas: la UNEME y la UVISA, la Unidad de Vida Saludable para pacientes que no tienen ninguna complicación [...] [entraba] un porcentaje bajo porque había que cumplir muchas condiciones para entrar al programa. Porque, para empezar, el diagnostico tenía que ser reciente, con un máximo de cinco años del diagnóstico sin complicaciones de ningún tipo. Es decir, o sea, si el paciente tenía un año, pero ya era diagnosticado con nefropatía, salía del programa... (Médico general, hombre)

La ausencia de equipos multidisciplinarios se señala como una barrera sobre la que no existe suficiente apoyo institucional ${ }^{(35)}$. Existen grupos en las instituciones, como el Programa de Atención al paciente Diabético DiabetIMSS, las Unidades de Especialidades Médicas (UNEME) y la Unidad de Visa Saludable (UVISA) en la cual se proporciona apoyo multidisciplinario para el paciente diabético. Pero estos apoyos son claramente insuficientes para la demanda existente. Por otro lado, en muchos casos, el equipo 
multidisciplinario no está completo y faltan elementos importantes, como el psicólogo, que puede ser indispensable para superar algunas de las barreras que el paciente presenta a la insulinización ${ }^{(25)}$. Aunque los entrevistados no plantean explícitamente que esta sea una barrera que les impida recomendar la terapia de insulinización son conscientes de que la educación del paciente podría continuar fuera del consultorio mediante la intervención de este equipo y, con su apoyo, sería factible enseñar a más pacientes cómo utilizar correctamente la insulina para que sea considerada como una opción terapéutica precoz.

\section{Desabasto de insumos necesarios para la terapia}

Este problema delicado es general en México, como lo muestran los trabajos consultados $^{(33,35)}$. Pero, como se mencionó antes, la situación económica del estado de Veracruz y de México como país ha conducido a situaciones de desabasto que se agravaron en la época en que esta investigación se concluía. Ello produjo graves dificultades para mantener las terapias con insulina ya que, en caso de que la institución no pueda proporcionar los insumos necesarios, muchos pacientes no estaban en condiciones económicas de hacerlo:

...ni siquiera soñaba en pasar a insulina con los pacientes, porque no teníamos refrigerador para la insulina. Teníamos que andarnos moviendo de comunidad en comunidad, y era imposible para mí transportar la insulina para los pacientes. Entonces eso me dificultaba completamente el pasar de un modelo a otro, ¿no?... (Médico general, hombre)

...institucionalmente hablando, nos quitaron del cuadro básico muchos medicamentos, no solamente antidiabéticos o insulina. Entonces eso nos limita a nosotros en el actuar. Si yo solamente dispongo de NPH, pues solamente puedo dar NPH, no puedo hacer más. Y sí, definitivamente influye mucho. Quizás no en la elección, sino influye más bien en el no adecuado control del paciente. Porque si yo convenzo a mi paciente $y$ lo tengo con NPH y requiere otras, otro tipo de insulina y no lo hay, me amarran las manos ¿no? (Médica familiar, mujer)

Finalmente, las barreras encontradas pueden ser esquematizadas de acuerdo con la gráfica presentada en la Figura 2. El esquema propuesto, con la organización de las barreras puede ser considerado como una aportación del presente trabajo; no tanto como esquema de clasificación o explicación, sino por el valor heurístico que pudiera tener para guiar intervenciones, ya que la caracterización de las barreras puede coadyuvar con intentos de solución o atenuación. Las barreras específicas (cuadros con líneas punteadas) se agruparon de acuerdo con su origen. Las barreras pueden originarse en situaciones propias del médico, obstáculos debidos a la organización institucional, y barreras surgidas en la relación médico paciente (cuadros intermedios). Todas estas temáticas son aspectos particulares del constructo más general de barreras a la insulinización.

Las barreras originadas en el propio personal pueden solucionarse fomentando programas educativos o intervenciones terapéuticas centrados en el médico. En cambio, los obstáculos institucionales requieren financiamiento, reorganización de las instituciones o formación de equipos multidisciplinarios. Las dificultades de la relación médico-paciente tendrían un enfoque educativo en ambos: en el médico, con el propósito de dotarlo de estrategias que posibiliten un lenguaje comprensible para el paciente, desarrollar empatía o habilidades de comunicación; y, para el paciente, diseñar talleres que coadyuven a sus estrategias de comprensión de la enfermedad, el tratamiento o las indicaciones del médico.

\section{Algunas posibles soluciones propuestas por el personal médico}

Un resultado inesperado pero muy importante fue que, en el transcurso de la 


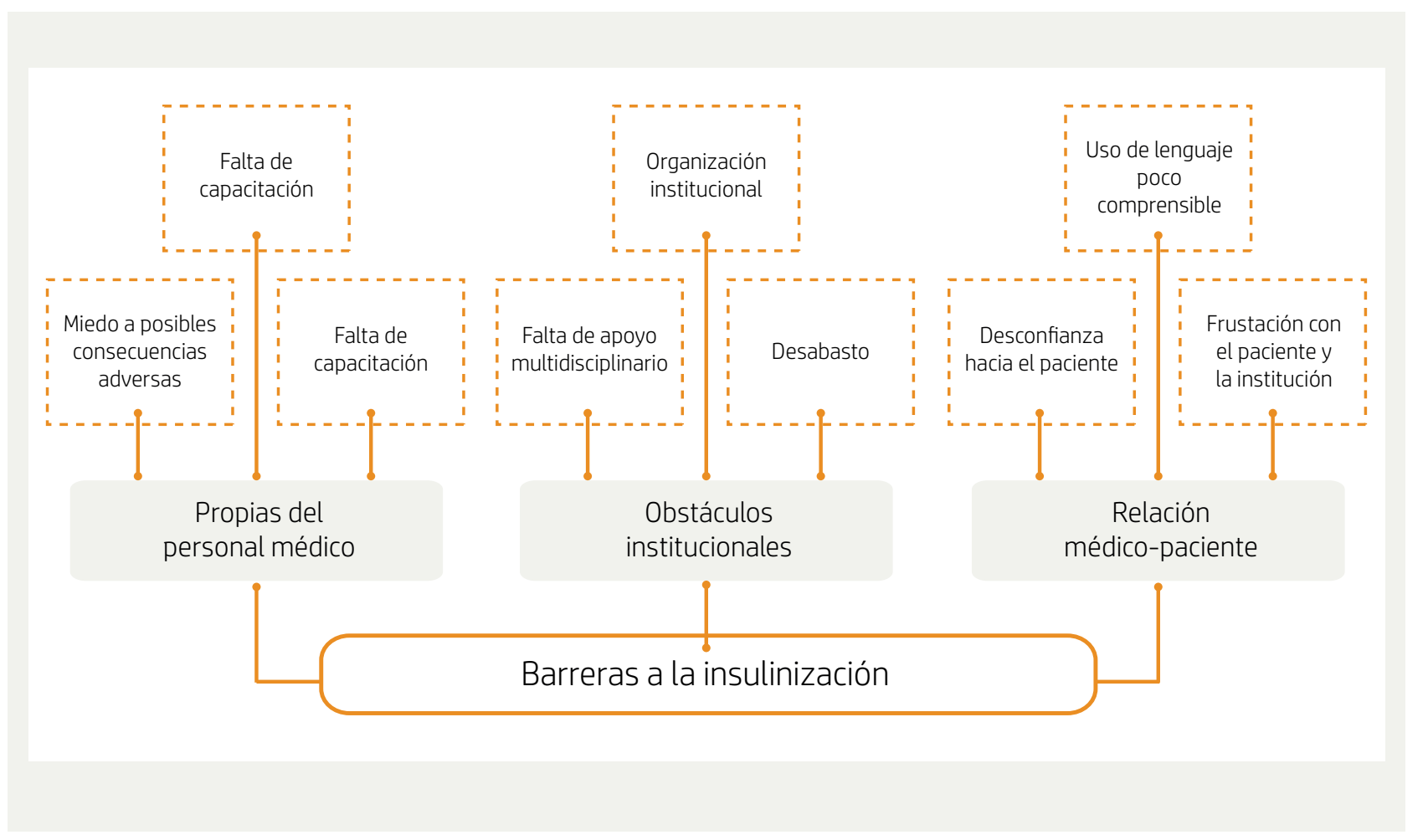

Figura 2. Esquema descriptivo de las barreras a la insulinización reportadas por los participantes del estudio. Ciudad de Xalapa, Veracruz, México, 2015 y 2016.

Fuente: Elaboración propia.

entrevista, casi la mitad de los médicos hablaron de posibles soluciones para algunas de estas barreras. De manera específica, las propuestas de solución consistían en: 1) la formación de equipos multidisciplinarios para atender a los pacientes, 2) actualización continua del personal, incluyendo la capacitación en educación y sensibilización de paciente, 3) presencia de un psicólogo de la salud en el equipo, 4) potenciar la confianza que el paciente suele crear con su médico, y 5) promover el uso de la insulinización temprana como alternativa terapéutica para los pacientes.

\section{Formación de equipos multidisciplinarios para la atención al paciente}

Las experiencias o la bibliografía consultada por los médicos les permiten plantear que la formación de equipos multidisciplinarios permitiría vencer barreras de los pacientes y los proveedores de salud, así como optimizar los recursos de las instituciones y mejorar la organización institucional, disminuyendo los tiempos de consulta al evitar que el médico duplique funciones que pueden realizar otros miembros del equipo de salud.

...tuve oportunidad de interactuar con un equipo multidisciplinario de diabetes cuando se hizo la prueba piloto del diabetIMSS, en el que el médico familiar nada más iba a dar consulta después de que, en la misma sala de espera, psicología, nutrición, y trabajo social hicieran su parte de promover un tema, y que la enfermera los pesara, los midiera, contestara alguna duda básica, y ya. A la hora de que el paciente acudiera con el médico, él nada más lo ajustaría al tratamiento o resolvería alguna duda extra. Este sistema multidisciplinario se evaluó 
a 18 meses. Los pacientes disminuyeron considerablemente su hemoglobina. Se demostró que el abordaje multidisciplinario pues era excelente para ellos. $Y$ entre lo multidisciplinario, a mí lo que más me quedó claro, la estrella de lo multidisciplinario era el aspecto psicológico. El empoderamiento que podían tener los pacientes, el traspasar esas barreras de negación y todo eso... (Médico general, hombre)

La experiencia positiva que mostraron los que interactuaron con el grupo multidisciplinario queda matizada con la necesidad de que los miembros del equipo respeten sus áreas de intervención, sus conocimientos y el objetivo común del equipo en beneficio de los pacientes.

\section{Actualización continua del personal de salud}

Los participantes enfatizaron la necesidad de la capacitación contante para estar al tanto de los nuevos tratamientos y tendencias (como la insulinización temprana), adquirir habilidades y competencias que no pudieron durante su formación, entre las cuales destacan la comunicación con el paciente:

...egresas de un posgrado por ejemplo y que eso no termina ahí. Tú necesitas estar en constante actualización, de ahí que constantemente hay jornadas, hay simposios, hay congresos, te inscribes a revistas, tienes que estar al tanto de todo lo que va surgiendo [...] Tienen que prepararse. El mismo instituto ofrece ese tipo de cursos, les da oportunidad de hacer profesionalización docente, de acudir a cursos monográficos para el manejo de la relación médico paciente, cursos de actualización en diabetes, curso de manejo de insulina para todos los médicos que están laborando en el instituto y que quieran acceder al curso. Entonces, las posibilidades si las hay... (Médica familiar, mujer)

\section{Presencia de un psicólogo de la salud en el equipo}

De manera interesante, algunos especialistas y médicos generales que habían tenido la oportunidad de interactuar en equipos multidisciplinarios, resaltaban la necesidad de contar con un psicólogo de la salud en el equipo, especialmente, en lo que se refería al manejo de conflictos y barreras, frustración del personal médico, y el empoderamiento y responsabilización del paciente por su enfermedad.

...tienen que definitivamente entrar...el área de la psicología, el área de la salud mental. ¿A consecuencia de qué? Yo creo que en Latinoamérica pensamos que ese personal es para gente que está mal de la cabeza, cuando realmente tienen una enorme gama de cosas en las que puede actuar ¿no? Realmente grande. Yo creo que ese sería el primer paso para que un paciente pudiera atender su enfermedad. Aceptar su enfermedad y ya después hablar de la insulinización, de la aceptación de todas esas situaciones... (Médico general, hombre)

...Creo que a veces uno mismo como médico lo necesita ¿no? Son tantas situaciones, tanta presión y dificultades... El famoso síndrome de burnout. Necesitamos técnicas para aliviar el estrés, para tener comunicación con nuestros pacientes, para no sentirnos tan frustrados. Son cosas que el psicólogo sabe y nosotros no... (Médica familiar, mujer)

\section{Potencializar la confianza que el paciente suele crear con su médico}

Algunos entrevistados tienen en claro que la relación del paciente con el médico puede contribuir a vencer barreras y lograr los objetivos terapéuticos y el empoderamiento del paciente, siempre que haya respeto mutuo y el médico tenga las habilidades para lograr una comunicación eficaz. 
...cuando un paciente llega contigo es porque tiene la confianza de que le vas a ayudar y eso ya es algo ganado. El piensa que tú tienes la capacidad de ayudarle, entonces hay que aprovechar esa parte para ofrecer, para explicar, para que el paciente comprenda y acepte; $y$, entonces, haga ese cambio que es necesario en él para estar mejor. La responsabilidad que tiene él es de cuidarse, de entender el proceso y de llevarlo a cabo. (Médica familiar, mujer)

\section{Promover el uso de la insulinización temprana como alternativa terapéutica}

Por último, los médicos consideran que el inicio temprano de la insulinización de pacientes seleccionados no solo mejoraría la calidad de vida de estos, sino que permitiría importantes ahorros a mediano y largo plazo para la institución y el sistema de salud.

...las insulinas pues sí, tienen un costo elevado relativamente. Sin embargo, si nosotros podemos visualizar a un futuro, el que el paciente esté controlado nos evita otro tipo de gastos. De hospitalización, cama, cirugía, gastos de las complicaciones, gastos de curaciones y todo lo que nos conlleva un mal control. Entonces, si somos visionarios, ese aspecto, no es que nos cueste más si manejamos insulinas, porque se puede llevar un adecuado control y con esto limitar un daño terciario... (Médica internista, mujer)

\section{DISCUSIÓN}

\section{Algunas posibilidades de interacción entre las barreras encontradas}

Del análisis de los fragmentos de discurso presentados se desprende que las barreras no actúan en forma aislada. A continuación, se presentan posibilidades de interacción entre las barreras que pueden ser tratadas en investigaciones posteriores.
Parece claro que los obstáculos institucionales influyen de manera importante, tanto sobre los médicos, como sobre la relación médico paciente. Los breves tiempos asignados a cada consulta, además de incrementar los sentimientos de frustración del médico, pueden condicionar la claridad del lenguaje con el cual se dirige al paciente y la educación que le debe proporcionar (ambos aspectos de la relación médico-paciente). Dado que los esquemas de tratamiento con insulina son complejos y requieren una cuidadosa capacitación del paciente, un médico bien entrenado, con amplia experiencia clínica, quizá podría encontrar con mayor facilidad las palabras adecuadas para obtener una comunicación efectiva, pero hemos visto anteriormente que los médicos generales y con menos experiencia tienen dificultades para lograr esto. Otros aspectos institucionales, como la falta de apoyo multidisciplinario pueden tener efectos similares; pues, en los fragmentos de discurso presentados, se puede apreciar que el concurso de un equipo multidisciplinario podría abreviar los tiempos de consulta (obstáculo institucional), proporcionar la información que no puede brindar el médico debido a su falta de capacitación (obstáculo propio del personal médico), y contribuir a mejorar la comunicación médico-paciente, coadyuvando a resolver dudas y dando capacitación adicional en el manejo del esquema terapéutico.

El desconocimiento del médico respecto de los esquemas de tratamiento con insulina, por sí mismo, puede hacer que ni siquiera se plantee la posibilidad del uso de la insulina. Pero cuando este se une a dificultades institucionales, pueden privar al paciente de cualquier posibilidad de recibir este tipo de terapia; incluso, en los casos en los que, de acuerdo con las guías de tratamiento actual, esto sería indispensable para su control adecuado. Esto ocurriría, por ejemplo, cuando un médico consciente de su desconocimiento necesita canalizar al paciente a un segundo nivel de atención, pero este nivel no existe en la clínica de adscripción, y el paciente no tiene los medios para acudir a los hospitales que sí lo tienen. El desabasto de 
los insumos necesarios para la terapia tendría efectos similares, y el paciente puede llegar a percibir que sus malestares aumentan y no está recibiendo la atención adecuada.

\section{El problema de la adherencia terapéutica}

La adherencia terapéutica es un posible punto de interacción entre las barreras encontradas que merece ser tratado aparte. Este término abarca tanto el cumplimiento de las indicaciones terapéuticas, como la persistencia en Ilevarlas a cabo desde el inicio hasta el final del tratamiento prescrito ${ }^{(51)}$. En una revisión sistemática, Asche et al. encontraron que la adherencia al tratamiento de la DM2 mejora el control de los niveles de glucosa, previene complicaciones, disminuye la utilización de los servicios de salud (como la hospitalización y las visitas al departamento de urgencias), disminuye la necesidad de pasar a regímenes de tratamiento más intensos, y hay algunas evidencias de que podría influir en la calidad de vida y disminuir los costos del cuidado de la salud de los pacientes ${ }^{(51)}$.

Entre los factores que contribuyen a que los pacientes no se adhieran al tratamiento están algunos de los que han surgido en la presente investigación. De manera destacada, se señalan los problemas de comunicación, la falta de comprensión por parte del médico de los problemas que tiene el paciente para afrontar la terapia, y la falta de capacidad para ayudarlo a encontrar soluciones que puedan implementar para persistir en la terapia ${ }^{(52)}$. Parece posible que la falta de capacitación del médico, la ausencia de apoyo interdisciplinario y el uso de un lenguaje no adecuado tengan una influencia importante en el surgimiento de estos problemas. Un médico no capacitado puede no percibir que existen esos problemas; $y$, si lo hace, puede que no sepa cómo solucionarlos. Y si no existen profesionales de otras áreas que puedan proporcionar el apoyo necesario, estas dificultades quedarán sin solución y podrían afectar la adherencia del paciente.
Lo mismo puede ocurrir con respecto a los factores atribuibles a los médicos que han demostrado favorecer la adherencia terapéutica. Entre los que tienen un efecto mayor se encuentran: la escucha activa, proveer soporte emocional, potenciar la confianza del paciente en su médico, dar información clara y suficiente, dar a cada paciente el tiempo necesario para su atención, identificar las barreras y preocupaciones del paciente con respecto a la terapia, hacer que el paciente colabore en la toma de decisiones respecto de la terapia, utilizar estrategias que mejoren la comunicación entre el médico y el paciente, y ayudar al paciente de bajos ingresos a encontrar alternativas para solventar el costo de la terapia ${ }^{(52,53,54,55,56)}$. Parece claro que los obstáculos institucionales pueden impedir dar a los pacientes el tiempo necesario, coadyuvar a que no puedan recibir su medicación, y que no se pueda dar el apoyo que el médico no está capacitado para dar. La escucha activa, el apoyo y la empatía que pueda proporcionar el médico dependen de que esté debidamente capacitado, de que exista una comunicación efectiva sin ambigüedades, y que la desconfianza y la frustración no obstaculicen estos procesos.

En resumen, el estudio de la adherencia terapéutica en la DM2 parece ser un campo adecuado para comprobar la influencia de las barreras detectadas en el presente estudio y estudiar su interacción.

\section{CONCLUSIONES}

El esquema propuesto muestra con claridad que las diferentes barreras a la insulinización temprana del personal médico proceden de diferentes fuentes y situaciones: 1) las barreras propias del personal médico, que proceden de su formación, su capacitación, sus conocimientos respecto a la terapéutica temprana con insulina, y el miedo a las posibles consecuencias adversas al tratamiento; 2) las barreras surgidas en la relación médico paciente, que se manifiestan en la desconfianza del médico y su frustración con 
el paciente, y el uso de un lenguaje poco comprensible; y 3) los obstáculos institucionales, destacando entre estos el desabasto de los insumos necesarios, la organización institucional desfavorable, y la falta de apoyo multidisciplinario.

Consideramos que el esquema tiene valor heurístico como propuesta de investigación y, todavía más importante, como esquema de manejo, control, y para generar propuestas de solución. Una manera de utilizarlo sería tomarlo como base para el análisis de la problemática en una institución determinada, detectar cuáles de las barreras se están presentando, junto con sus interacciones y sus posibles estrategias de solución.

Los hallazgos de esta investigación podrían dar pie a diversas estrategias de intervención en profesionales y estudiantes de medicina, con el objetivo de reflexionar en torno a los aspectos que, desde su práctica, podrían favorecer una relación con sus pacientes centrada en las necesidades y características sociales, culturales y psicológicas de cada uno de ellos. En este sentido, un aspecto angular sería considerar la importancia de diferenciar entre las enfermedades como entidades clínicas de los padecimientos como experiencias subjetivas ${ }^{(57)}$.

Las barreras propias del personal pueden ser abordadas mediante cursos de capacitación o subespecialización, pero las que se generan en la relación con el paciente pueden requerir una intervención por parte de otros miembros del personal de salud. Las situaciones de desabasto y reorganización institucional van más allá del dominio del personal de salud, y requieren la intervención de autoridades, administradores y visión a mediano y largo plazo para encontrar soluciones que, aunque puedan ser costosas a corto plazo, permitirían ahorros sustanciales al sistema de salud en el futuro.

Algunos de los hallazgos de este trabajo son similares a los reportados en la bibliografía mundial, pero se evidencia claramente que las dificultades económicas, administrativas y la idiosincrasia propia del paciente y el personal médico de México subrayan la importancia de las situaciones de capacitación, obstáculos institucionales y deficiencias de educación de los pacientes y el personal de salud.

Por último, aunque el esquema propuesto no pretende ser exhaustivo, consideramos que puede actuar como punto de partida para investigaciones en otras regiones o países. Los diversos abordajes cualitativos, cuantitativos y mixtos permitirán plantear un panorama realista y útil que, modificando o reforzando el esquema propuesto, permita la comprensión y resolución de las barreras a la terapia de insulinización temprana en la realidad investigada.

\section{AGRADECIMIENTOS}

El presente estudio fue financiado, en parte, mediante un Apoyo a la Incorporación de Nuevos Profesores de Tiempo Completo del sistema PRODEP del Gobierno Federal de México (folio: UV-PTC-793). Los autores desean agradecer a los doctores Jaime Morales Romero, del Instituto de Salud Pública de la Universidad Veracruzana, y Jorge Iván Zurutuza Lorméndez por sus atenciones y generoso apoyo para la consecución de los fines del presente estudio.

\section{REFERENCIAS BIBLIOGRÁFICAS}

1. Escobar MC. Mitos sobre la prevención de las enfermedades no transmisibles en América Latina. Salud Pública de México. 2000;42:56-64.
2. American Diabetes Association. Diagnosis and Classification of Diabetes Mellitus. Diabetes Care. 2014;37(Suppl 1):S81-S90.

3. World Health Organization. Diet, nutrition and the prevention of chronic diseases. (World Health Organ Technical Report Series No. 916). Washington: $\mathrm{WHO} ; 2003$.

4. Organización Mundial de la Salud. Informe mundial sobre diabetes: Resumen de orientación [Internet]. 2016 [citado 19 ene 2017]. Disponible en: https://goo.gl/DYwZs7.

5. Hernández-Ávila M, Gutiérrez J, ReynosoNoverón N. Diabetes mellitus en México: El estado de la epidemia. Salud Pública de México. 2013;55(Suppl 1):S129-S136. 
6. Villalpando S, De la Cruz V, Rojas R, ShamahLevy T, Ávila MA, Gaona B, Rebollar R, Hernández L. Prevalence and distribution of type 2 Diabetes mellitus in Mexican adult population: A probabilistic survey. Salud Pública de México. 2010;52(Suppl 1):S19-S26.

7. Rull JA, Aguilar-Salinas CA, Rojas R, Ríos-Torres JM, Gomez-Perez FJ, Olaiz G. Epidemiology of type 2 diabetes in México. Archives of Medical Research. 2005;36:188-196.

8. García-Soto CE. El costo de vivir con diabetes. Brújula de Compra [Internet]. 2007 [citado 19 ene 2017]. Disponible en: https://goo.gl/BL13cJ.

9. Gómez-Dantés O, Sesma S, Becerril VM, Knaul $\mathrm{F}$, Arreola H, Frenk J. Sistema de salud en México. Salud Pública de México. 2011;53(Supl 2):S220S232.

10. Instituto Nacional de Estadística, Geografía e Informática. Número de habitantes de Veracruz [Internet]. 2015 [citado 19 ene 2017]. Disponible en: https://goo.gl/U1g4QR.

11. Standards of Medical Care in Diabetes. Glycemic targets. Diabetes Care. 2016;39(Suppl 1):S39-S46.

12. Nathan DM, Buse JB, Davidson MB, Ferrannini E, Holman RR, Sherwin R, Zinman B. Medical management of hyperglycaemia in type 2 diabetes mellitus: a consensus algorithm for the initiation and adjustment of therapy. Diabetologia. 2009;52:17-30.

13. Menéndez TE, Lafita TFJ, Artola MS, Millán NCJ, Alonso GA, Puig DM, García SJR, Álvarez GF, García AJ, Mediavilla BJ, Miranda FC, Romero GR. Recomendaciones para el tratamiento farmacológico de la hiperglucemia en la diabetes tipo 2. Endocrinology and Nutrition. 2011;58:112-120.

14. National Institute for Health Care Excellence. Algorithm for blood glucose lowering therapy in adults with type 2 diabetes [Internet]. 2015 [citado 23 may 2017]. Disponible en: https://goo.gl/ QmjHY8.

15. Inzucchi SE, Bergenstal RM, Buse JB, Diamant M, Ferrannini E, Nauck M, Peters AL, Tsapas A, Wender R, Matthews DR. Management of hyperglycaemia in type 2 diabetes, 2015: a patientcentred approach; update to a position statement of the American Diabetes Association and the European Association for the Study of Diabetes. Diabetología. 2015;58(3):429-442.

16. Asociación Latinoamericana de Diabetes. Guías ALAD sobre el diagnóstico, control y tratamiento de la Diabetes Mellitus tipo 2 con medicina basada en evidencia [Internet]. 2013 [citado 23 may 2017]. Disponible en: https://goo.gl/RE8LVu.

17. Müssig K. [Insulin therapy: as early as possible]. Deutsche Medizinische Wochenschrift. 2016;141(8):578-579.

18. Hanefeld $M$, Bramlage P. Insulin use early in the course of type 2 diabetes mellitus: the ORIGIN trial. Current Diabetes Reports. 2013;13:342-349.

19.- Sorli C. Insulin therapy in type 2 diabetes: a reflection on the state of the art today, and the potential journeys yet to come. American Journal of Medicine. 2014;127(Suppl 1):S1-S2.

20. Lovre D, Fonseca D. Benefits of timely basal insulin control in patients with type 2 diabetes. Journal of Diabetes Complications. 2015;29:295301.

21. Lerman I, Moreira-Díaz JP, Romero-Ibarguengoitia ME, Gómez-Pérez FJ. Reluctance to insulin therapy in low-income, type 2 diabetic patients: the need for stronger reinforcement strategies. Endocrine Practice. 2009;15:41-46.

22. Vinagre I, Conget I. Control of type 2 diabetes mellitus in Spain today: identification of the main obstacles in daily clinical practice. Medicina Clínica. 2013;144(Suppl 2):S3-S6.

23. Nathan DM. Initial management of glycemia in type 2 diabetes mellitus. New England Journal of Medicine. 2002;347:1342-1349.

24. Meece J. Dispelling myths and removing barriers about insulin in type 2 diabetes. The Diabetes Educator. 2006;32(Suppl 1):S9-S18.

25. Peyrot $M$, Rubin RR, Lauritzen $T$, Skovlund SE, Snoek FJ, Matthews DR, Landgraf R, Kleinebreil L, International DAWN Advisory Panel. Resistance to insulin therapy among patients and providers: results of the cross-national Diabetes Attitudes, Wishes, and Needs (DAWN) study. Diabetes Care. 2005;28(11):2673-2679.

26. UK Prospective Diabetes Study (UKPDS) Group. Intensive blood glucose control with sulfonylureas or insulin compared with conventional treatment and risk of complications in patients with type 2 diabetes (UKPDS 33). Lancet. 1998;352:837-853.

27. Polonsky WH, Fisher L, Guzmán S, Villa-Caballero L, Edelman, SV. Psychological insulin resistance in patients with type 2 diabetes: the scope of the problem. Diabetes Care. 2005;28:25432545. 
28. Hunt LM, Pud A, Valenzuela M, Ba A, Pugh, J. NIDDM Patients' Fears and Hopes About Insulin Therap. Diabetes Care. 1997; 20: 294-298.

29. Karter AJ, Parker MM, Swain BE, Moffet $\mathrm{HH}$, Subramanian $U$, Saha C. Barriers to Insulin Initiation. The Translating Research Into Action for Diabetes Insulin Starts Project. Diabetes Care. 2010;33:733-735.

30. Bogatean MP, Hancu N. People with type 2 diabetes facing the reality of starting insulin therapy: factors involved in psychological insulin resistance. Practical Diabetes International. 2004;21:247-252.

31. Oliveria SA, Menditto LA, Yood MU, Koo YH, Wells KE, McCarthy BD. Barriers to the initiation of, and persistence with, insulin therapy. Current Medical Research and Opinion. 2008;23:31053112.

32. Lee YK, Lee PY, Ng CJ. A qualitative study on healthcare professionals' perceived barriers to insulin initiation in a multi-ethnic population. BMC Family Practice. 2012;13:28. Doi: 10.1186/14712296-13-28.

33. Lerman I. Barreras que dificultan la aplicación temprana de insulina en el paciente con diabetes tipo 2. ALAD Asociación Latinoamericana de Diabetes. 2009;XII(2):66-68.

34. Polinski J, Smith B, Curtis B, Seeger J, Choundhry N, Connolly J, Shrank W. Barriers to insulin progression among patients whit type 2 diabetes: a systematic review. Diabetes Educator. 2013;39(1):53-65.

35. Ávalos-García M, López-Ramón C, Priego-Álvarez $\mathrm{H}$, Córdova-Hernández A, Morales-García M. Barreras y perspectivas del personal de salud en el control de la diabetes mellitus en unidades médicas de la seguridad social de Tabasco: Un estudio cualitativo. Horizonte Sanitario. 2013;12(3):111-119.

36. Estados Unidos Mexicanos. Norma Oficial Mexicana NOM 015-SSA2-2010 Para la la prevención, el control y el tratamiento de la diabetes mellitus [Internet]. 2010 [citado 6 feb 2017]. Disponible en: https://goo.gl/TGyuSw.

37. Subsecretaría de Integración y Desarrollo del Sector Salud. Modelo de atención integral de salud (MAI): Documento de arranque [Internet]. 2015 [citado 6 feb 2017]. Disponible en: https:// goo.gl/xshJgX.

38. Jiménez-Corona A, Aguilar-Salinas CA, RojasMartínez R, Hernández-Ávila M. Diabetes Mellitus tipo 2 y frecuencia de acciones para su prevención y control. Salud Pública de México. 2013;55(Supl 2):S137-S143.

39. Álvarez-Gayou JJL. Cómo hacer investigación cualitativa: Fundamentos y metodología. México: Paidós Educador; 2003.

40. Martínez-Salgado C. El muestreo en investigación cualitativa: principios básicos y algunas controversias. Cïncia \& Saúde Coletiva. 2012;17(3):613-619.

41. Graneheim UH, Lundman B. Qualitative content analysis in nursing research: concepts, procedures and measures to achieve trustworthiness. Nurse Education Today. 2004;24:105112.

42. Hernández SR, Fernández CC, Baptista LP. Metodología de la investigación. 6a ed. México: Mc Graw Hill Education; 2014.

43. Eldeman S, Pettus J. Challenges associated with insulin therapy in type 2 diabetes mellitus. American Journal of Medicine. 2014;127(Suppl):S11S16.

44. Peyrot M, Rubin RR, Khunti K. Adressing barriers to initiation of insulin in patients whit type 2 diabetes. Primary Care Diabetes. 2010;4(Suppl 1):S11-S18.

45. Lara y Mateos RM. Medicina y cultura: hacia una formación integral del profesional de la salud. 2a ed. México: El Manual Moderno; 2005.

46. Barsky AJ. The paradox of health. The New England Journal of Medicine. 1988;318(7):414418.

47. Castro R. La vida en la adversidad: el significado de la salud y la reproducción en la pobreza. México: CRIM/UNAM; 2000.

48. Trujano R, Vega Valero Z, Nava Quiroz C, Saavedra Vásquez K. Interacción médico-paciente y su relación con el control del padecimiento en enfermos crónicos. Liberabit. 2011;17(2):223-230.

49. Escudero-Carretero MJ, Prieto-Rodríguez $M$, Fernández-Fernández I, March-Cerdà J. La relación médico-paciente en el tratamiento de la diabetes tipo 1: Un estudio cualitativo. Atención Primaria. 2006;38(1):8-18.

50. Martín L, Grau J. La investigación de la adherencia terapéutica como un problema de la psicología de la salud. Psicología y Salud. 2004;14(1):89-99. 
51. Asche C, LaFleur J, Conner C. A review of diabetes treatment adherence and the association with clinical and economic outcomes. Clinical Therapy. 2011;33(1):74-109.

52. Brunton SA, Polonsky WH. Medication adherence in type 2 diabetes mellitus: real-world strategies for addressing a common problem. The Journal of Family Practice. 2017;66(Suppl 4):S46S51.

53. Hall MA, Dugan E, Zheng B, Mishra AK. Trust in physicians and medical institutions: what is it, can it be measured, and does it matter? Milbank Quarterly. 2001;79(4):613-639.

54. Keating NL, Gandhi TK, Orav EJ, Bates DW, Ayanian JZ. Patient characteristics and experiences associated with trust in specialist physicians. Archives of Internal Medicine. 2004;164(9):1015-1020.

55. Fiscella K, Meldrum S, Franks $P$, Shields CG, Duberstein P, McDaniel SH, Epstein RM. Patient trust: is it related to patient-centered behavior of primary care physicians? Medical Care. 2004;42(11):1049-1055.

56. Duberstein $P$, Meldrum S, Fiscella K, Shields CG, Epstein RM. Influences on patients' ratings of physicians: Physicians demographics and personality. Patient Education Counseling. 2007;65(2):270-274.

57. Cassel EJ. The Healer's Art: A new approach to the doctor-patient relationship. New York: Lippincot; 1976.

\section{FORMA DE CITAR}

Lagunes-Córdoba R, Galindo-Guevara I, Castillo Reyes A, Romero-Aparicio C, Rosas-Santiago FJ. ;Por qué los médicos no utilizan en forma temprana la insulinización en pacientes con diabetes mellitus tipo 2?: Un estudio cualitativo en una ciudad mexicana. Salud Colectiva. 2017;13(4):693-712. doi: 10.18294/sc.2017.1341

Recibido: 4 de marzo de 2017 | Versión final: 25 de abril de 2017 | Aprobado: 30 de mayo de 2017

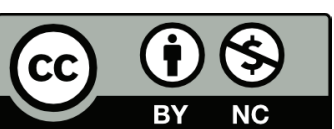

Este obra está bajo una licencia de Creative Commons Reconocimiento-NoComercial 4.0 Internacional. Reconocimiento - Permite copiar, distribuir y comunicar públicamente la obra. A cambio, se debe reconocer y citar al autor original. No Comercial - Esta obra no puede ser utilizada con finalidades comerciales, a menos que se obtenga el permiso. 